\title{
Excitation of emission lines by fluorescence and recombination in IC 418
}

\author{
Vladimir Escalante ${ }^{1}$, Cristophe Morisset $^{2 \dagger}$, and Leonid Georgiev ${ }^{2}$ \\ ${ }^{1}$ CRyA, UNAM, Ap. Postal 72-3, C. P. 58091, Morelia, Michoacán, México \\ ${ }^{2}$ Instituto de Astronomía, UNAM Ap. Postal 70-264, C. P. 04510, México, D. F., México \\ emails: v.escalante@crya.unam.mx, chris.morisset@gmail.com, georgiev@astro.unam.mx
}

\begin{abstract}
We predict intensities of lines of CII, NI, NII, OI and OII and compare them with a deep spectroscopic survey of IC 418 to test the effect of excitation of nebular emission lines by continuum fluorescence of starlight. Our calculations use a nebular model and a synthetic spectrum of its central star to take into account excitation of the lines by continuum fluorescence and recombination. The NII spectrum is mostly produced by fluorescence due to the low excitation conditions of the nebula, but many CII and OII lines have more excitation by fluorescence than recombination. In the neutral envelope, the NI permitted lines are excited by fluorescence, and almost all the OI lines are excited by recombination. Electron excitation produces the forbidden optical lines of OI, but continuum fluorescence excites most of the NI forbidden line intensities. Lines excited by fluorescence of light below the Lyman limit thus suggest a new diagnostic to explore the photodissociation region of a nebula.
\end{abstract}

Keywords. planetary nebulae: individual (IC 418), atomic processes, radiation mechanisms: general

\section{Models}

Morisset \& Georgiev (2009) demonstrated that is it possible to construct consistent models of the central star atmosphere and the nebula to predict accurately the intensities of UV, optical and IR nebular and stellar emission lines in IC 418. We used similar models and included excitation of dipole-allowed transitions by fluorescence besides recombination to predict the intensities of 189 emission lines of C II, N I, N II, O I, and O II observed by Sharpee et al. (2003).

The high-resolution synthetic spectrum produced by the CMFGEN code (Hillier \& Miller (1998)) gives the opportunity to notice variations in the predicted intensities of some emission lines due to the expansion of the nebular gas. We used the CLOUDY nebular code (Ferland et al. 1998) to model the nearly spherical nebula with a density profile similar to the one used by Morisset \& Georgiev (2009). Fluorescence is important in the population of $\mathrm{s}, \mathrm{p}$, and some $\mathrm{d}$ states while recombination populates $\mathrm{f}$ and $\mathrm{g}$ states.

\section{Results}

The most important effect of fluorescence is in the N II lines from the decay of $\mathrm{s}$, $\mathrm{p}$ and most $\mathrm{d}$ states. Intense lines in planetary nebulae like $3 \mathrm{~s}^{3} \mathrm{P}_{1}^{\mathrm{o}}-3 p^{3} D_{4} \lambda 5666.63$ and $3 \mathrm{~s}^{3} \mathrm{P}_{2}^{\mathrm{o}}-3 \mathrm{p}^{3} \mathrm{D}_{3} \lambda 5679.56$ have fluorescence contributions of $78 \%$ and $73 \%$ respectively. C II lines from most p and d states have between $20 \%$ and $69 \%$ contribution by fluorescence. O II lines are mostly excited by recombination, but fluorescence can contribute up to $50 \%$ of the excitation of $3 \mathrm{~d}^{2} \mathrm{~F}$ states (Figs. 1 and 2).

$\dagger$ Temporarily at Instituto de Astrofísica de Canarias, 38200, La Laguna, Tenerife, Spain. 

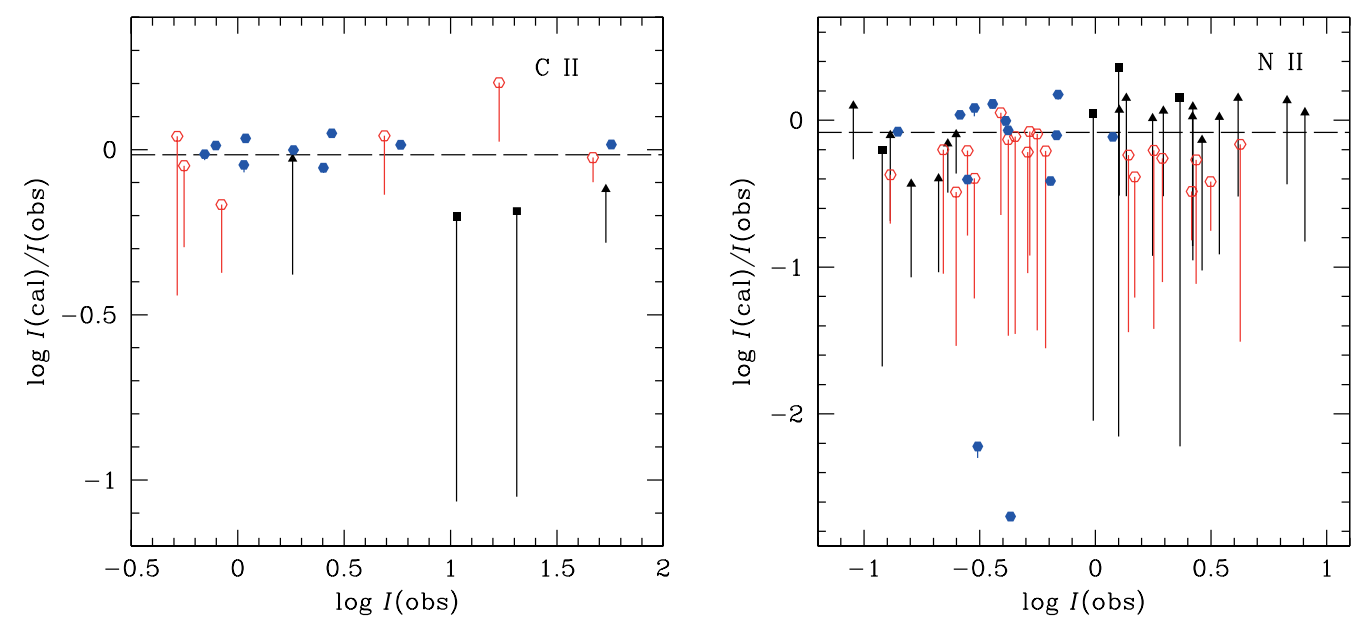

Figure 1. Comparison of calculated and observed line intensities of C II and N II. Lines from s states: black squares, p states: black triangles, $d$ states: red circles, $f$ and $g$ states: blue filled circles. The dashed line is the average $I$ (calc)/ $I$ (obs). Vertical lines show the increase of intensity due to fluorescence excitation (colour on line).
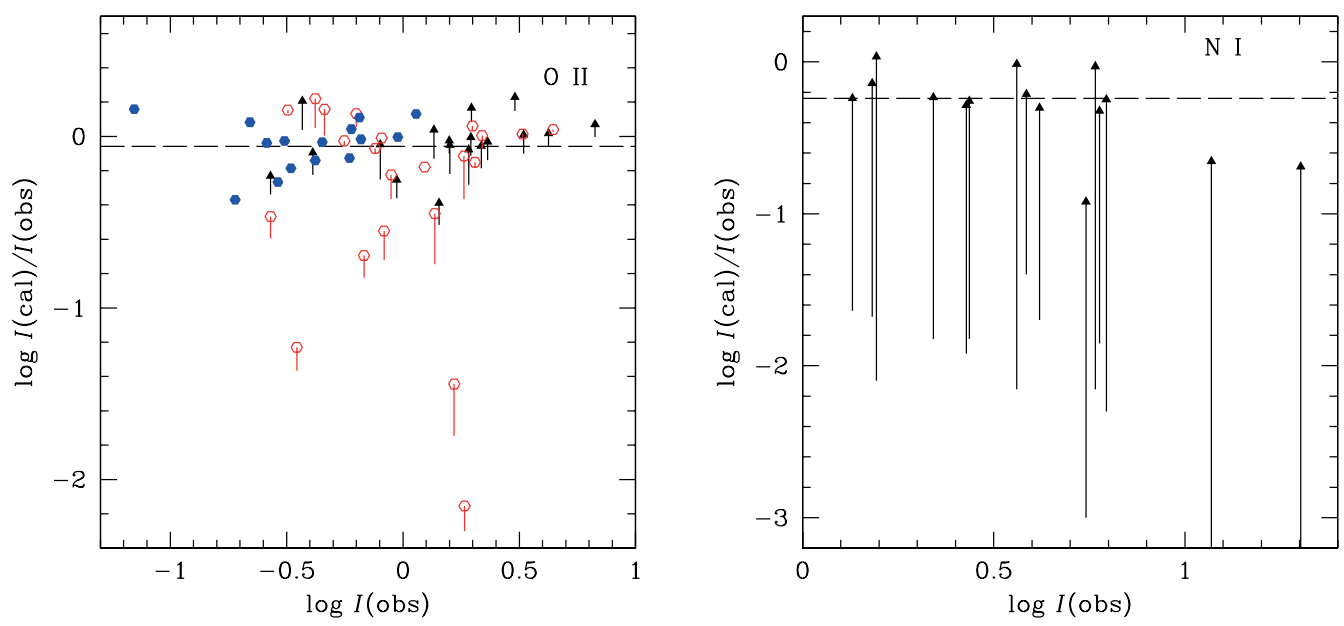

Figure 2. Same as figure 1 for O II and N I (colour on line).

We can also predict some of the intensities of the N I and O I in a neutral shell around the ionized region. Most permitted O I lines are produced by recombination, while N I permitted and optical forbidden lines are excited by fluorescence (Fig. 2).

\section{References}

Escalante, V. \& Morisset, C. 2005, MNRAS, 361, 813

Ferland, G. J., Korista, K. T., Verner, D. A., Ferguson, J. W., Kingdon, J. B., \& Verner, E. M., 1998, PASP, 110, 761

Hillier, D. J. \& Miller, D. L. 1998, ApJ, 496, 407

Morisset, C. \& Georgiev, L. 2009, A\& A, 507, 1517

Sharpee, B., Williams, R., Baldwin, J. A., \& van Hoof, P. A. M. 2003, ApJS, 149, 157 